\title{
An Analytical Model of the Response of the Meridional Overturning Circulation to Changes in Wind and Buoyancy Forcing
}

\author{
CAllum J. Shakespeare AND ANDrew McC. HogG \\ Research School of Earth Sciences, and ARC Centre of Excellence for Climate System Science, \\ The Australian National University, Canberra, Australian Capital Territory, Australia
}

(Manuscript received 25 October 2011, in final form 4 April 2012)

\begin{abstract}
An analytical model of the full-depth ocean stratification and meridional overturning circulation for an idealized Atlantic basin with a circumpolar channel is presented. The model explicitly describes the ocean response to both Southern Ocean winds and the global pattern and strength of prescribed surface buoyancy fluxes. The construction of three layers, defined by the two isopycnals of overturning extrema, allows the description of circulation and stratification in both the upper and abyssal ocean. The system is fully solved in the adiabatic limit to yield scales for the surface layer thickness, buoyancies of each layer, and overturning magnitudes. The analytical model also allows scaling of the Antarctic Circumpolar Current (ACC) transport. The veracity of the three-layer framework and derived scales is confirmed by applying the analytical model to an idealized geometry, eddy-permitting ocean general circulation model.

Consistent with previous results, the abyssal overturning is found to scale inversely with wind stress, whereas the North Atlantic overturning and surface-layer thickness scale linearly with wind stress. In terms of the prescribed surface buoyancy fluxes, increased negative fluxes (buoyancy removal) in the North Atlantic increase the North Atlantic overturning and surface-layer thickness, whereas increased positive fluxes in the middle and low latitudes lead to a decrease in both parameters. Increased negative surface buoyancy fluxes to the south of Drake Passage increase the abyssal overturning and reduce the abyssal buoyancy. The ACC transport scales to first order with the sum of the Ekman transport and the abyssal overturning and thus increases with both wind stress and southern surface buoyancy flux magnitude.
\end{abstract}

\section{Introduction}

Surface buoyancy fluxes and mechanical forcing of the ocean generates stratification and mixing, and drives circulation over the entire depth of the ocean. Water masses are transformed and cycled through the ocean by the meridional overturning circulation (MOC), a largescale convection pattern consisting of two cells in the latitude-density plane, with the upper corresponding to North Atlantic sinking and the lower resulting from buoyancy loss and downwelling over the Antarctic continental shelf (e.g., Fig. 2 of Lumpkin and Speer 2007). The heat transport and ocean ventilation processes associated with the MOC imply that it plays an essential role in determining future climatic changes

Corresponding author address: Andrew McC. Hogg, Research School of Earth Sciences, and ARC Centre of Excellence for Climate System Science, The Australian National University, Canberra, ACT 0200, Australia.

E-mail: andy.hogg@anu.edu.au arising from increasing atmospheric concentrations of anthropogenic carbon dioxide (Rintoul et al. 2001). Quantitative prediction of climatic change, even to first order, thus requires an understanding of the response of the MOC to a given change in surface forcing conditions. One of the primary mechanisms of describing this response is through the construction of simple analytical models of the MOC.

Arguably the most common analytical model in the oceanographic literature is the two-layer pycnocline model of Gnanadesikan (1999, hereafter G99). ${ }^{1}$ G99 builds on many previous models (e.g., Samelson and Vallis 1997) in describing midocean diapycnal upwelling and North Atlantic sinking but with the important innovation of simultaneously representing Southern

\footnotetext{
${ }^{1}$ An alternative modeling approach to the same problem, recently introduced by Nikurashin and Vallis (2012), involves matching conditions in independent basin and channel oceans; this parallel development complements the results shown here.
} 
Ocean winds (following Toggweiler and Samelson 1995) and eddies, thus emphasizing the dual-hemisphere nature of the MOC and the importance of the Southern Ocean as a driver of the global circulation (Hallberg and Gnanadesikan 2006). However, the G99 model and its subsequent adaptations (e.g., the time-dependent version by Allison et al. 2011) neglect the lower (abyssal) overturning cell and study the upper (North Atlantic) cell in isolation. That is, the model does not represent the negative surface buoyancy fluxes-with negative implying buoyancy loss from the ocean-near the Antarctic continent (to the south of Drake Passage) that are primarily responsible (e.g., Gordon 2009) for Antarctic Bottom Water (AABW) formation as dense shelf water. This treatment of the upper cell as independent of the abyssal cell is highly questionable because the two are expected to interact through diapycnal fluxes in the ocean interior and Southern Ocean eddy and Ekman mass fluxes (Ito and Marshall 2008). Indeed, if the water mass of the abyssal cell only outcrops near Antarctica where the surface buoyancy fluxes are negative, then there must exist a buoyancy transfer from the upper cell to the abyssal cell to compensate for these buoyancy losses, presumably via the aforementioned mass fluxes. Thus, the two MOC cells are connected through a thermodynamic balance, something not considered in G99.

It is desirable that any analytical model of the MOC also correctly describes the Antarctic Circumpolar Current (ACC), which is closely linked to the MOC and its associated stratification by the thermal wind relation (Borowski et al. 2002; Hogg 2010). The ACC can also cause feedbacks to the MOC via its effect on the Southern Ocean mesoscale eddy field (Marshall and Radko 2003). However, formulation of a unified MOC-ACC model presents significant difficulties. For example, the single pycnocline depth scale of the G99 model leads to scalings for the ACC transport that are difficult to reconcile with other models, such as the prediction that it is only proportional to the North Atlantic sinking, with no dependence upon other buoyancy or volume fluxes (see Allison et al. 2011; Fučkar and Vallis 2007). Levermann and Griesel (2004) also question whether a single depth scale can describe all the processes parameterized by G99 and thus multiple depth scales appear to be necessary to support a fully unified model of the MOCACC system. de Boer et al. (2010) provide some insight into a possible alternative depth scale by demonstrating that the parameterization of the North Atlantic sinking in G99 is only correct if the depth scale employed is the "depth of maximum (North Atlantic) overturning" rather than the pycnocline depth. This result motivates the formulation of a model with layer interfaces given by the depths at which the overturning streamfunction (for each MOC cell) is maximized, rather than having layers based on the stratification.

The explicit representation of surface buoyancy fluxes is necessary to construct a predictive analytical model of the MOC. G99 implicitly include the effect of buoyancy fluxes via a reduced gravity parameter, but it is not clear how changing the surface buoyancy flux field will alter this parameter. In addition, the reduced gravity must have some dependence on the model pycnocline depth and thus treating it as a prescribed parameter can significantly reduce the veracity of the model (Levermann and Furst 2010). Even current models of the abyssal overturning cell (e.g., Ito and Marshall 2008; Nikurashin and Vallis 2011) do not resolve the effect of arbitrary variations in the surface buoyancy forcing on the ocean stratification. Given that the pattern and strength of ocean-atmosphere buoyancy fluxes are expected to vary with anthropogenic climate change, the lack of an explicit representation of surface buoyancy fluxes is a serious deficiency in most current analytical models. Johnson et al. (2007) present a box model based on G99 that partially resolves this flux problem by introducing heat and salt budgets (i.e., a thermodynamic balance) for each box, and modeling the surface buoyancy forcing by water fluxes and imposed surface temperatures. They are then able to deduce the density and circulation structure of the equilibrium solution from the forcing parameters. However, the model does not represent the ACC and nor do the solutions allow a dual-cell MOC.

The objective of this paper is to produce an analytical model of the unified MOC-ACC system describing both the upper and lower cells of the MOC. In section 2, we formulate a model based primarily on the abovesuggested modifications to the G99 model, as well as a thermodynamic balance in the spirit of Johnson et al. (2007). In section 3, we introduce an idealized, eddypermitting general circulation model to test the theory. The results of the analytical and numerical models are then compared in section 4.

\section{Analytical model}

We introduce a conceptual framework for the Atlantic MOC (see Fig. 1) with four key differences from previous analytical models such as the G99 model:

(i) The principal-layer height scales are the "depths of overturning extrema." That is, as motivated by de Boer et al. (2010), we choose motional depth scales rather than a pycnocline scale to construct the layered model.

(ii) The existence of two MOC cells results in three large-scale flows/layers: a northward abyssal flow of 


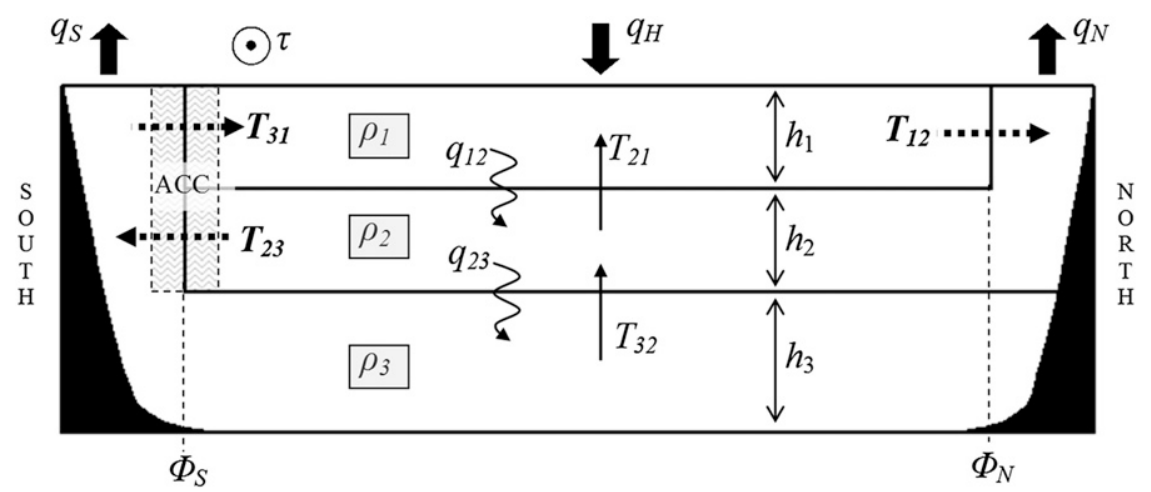

FIG. 1. The three-layer model of the Atlantic basin with the layers labeled as follows: surface layer (layer 1), middepth layer (layer 2), and abyssal layer (layer 3). The interlayer transports $T_{i j}$, layer densities $\rho_{i}$, and layer thicknesses $h_{i}$ are indicated. The model is forced by a Southern Ocean zonal wind stress $\tau$ and prescribed surface buoyancy fluxes $q_{S}, q_{N}$, and $q_{H}$, as shown. Buoyancy diffuses downward from layer 1 to layer 2, with flux $q_{12}$, and then from layer 2 to layer 3 , with flux $q_{23}$, as represented by the squiggly arrows. Positions $\phi_{S}$ and $\phi_{N}$ are the layer end latitudes, and $\phi_{S}$ lies over the shaded region representing the ACC passing through Drake Passage. In the adiabatic limit, the only nonzero fluxes are the transports $T_{12}, T_{23}$, and $T_{31}$ shown in boldface with thick dotted arrows. In this limit, they form a single flow pathway northward in layer 1 and southward in layer 2, with a stagnant abyssal layer.

dense water (layer 3), a southward middepth flow of intermediate density water (layer 2), and a predominantly northward surface flow of light water (layer 1).

(iii) The model is explicitly forced by a combination of prescribed surface buoyancy fluxes and Southern Ocean winds.

(iv) The densities of the three layers are considered as dependent variables.

Let us define the zonally summed overturning streamfunction as

$$
\psi(y, \rho, t)=-\iint_{-H(x, y)}^{z(x, y, \rho, t)} v\left(x, y, z^{\prime}, t\right) d z^{\prime} d x,
$$

where $v$ is the meridional velocity, $\rho$ is the in situ density, $H(x, y)$ is the ocean depth, and the zonal integral is over all $x$. The existence of two MOC cells implies that there are two isopycnals for which the meridionally and temporally averaged overturning streamfunction has local extrema. That is,

$$
\frac{\partial}{\partial \rho} \int \bar{\psi}(y, \rho) d y=0 \Rightarrow \rho=\rho_{L}, \rho_{U} \quad \text { with } \quad \rho_{L}>\rho_{U},
$$

where the overbar denotes a temporal mean and the meridional integral is over a suitable range of latitudes. The layer from the ocean bottom up to the isopycnal interface $\rho_{L}$ [or depth $h_{L}(x, y, t)=-z\left(x, y, t, \rho_{L}\right)$ ] is the abyssal layer (layer 3 ), and the above definition implies that the northward transport in this layer [i.e., the abyssal overturning magnitude, $\psi=-\psi\left(\rho=\rho_{L}\right)$ ] is maximized. The layer from isopycnal interface $\rho_{U}$ [or depth $\left.h_{U}(x, y, t)=-z\left(x, y, t, \rho_{U}\right)\right]$ up to the ocean surface is the surface layer (layer 1 ). The above definition implies that the northward transport in this layer [i.e., the North Atlantic overturning magnitude $\left.\psi=\psi\left(\rho=\rho_{U}\right)\right]$ is maximized. The layer between the two isopycnal interfaces is the middepth layer (layer 2) and the above definition implies that the southward flow in this layer is maximized.

Thus, we have three clearly defined layers separated by isopycnal interfaces $\rho_{L}$ and $\rho_{U}$ which we term the "isopycnals of overturning extrema" (IOEs). The layer thicknesses $h_{i}$ can be obtained by averaging over the entire surface area of the model ocean, denoted by \langle\rangle . For example,

$$
\begin{aligned}
h_{2} & =\left\langle\bar{h}_{L}(x, y)-\bar{h}_{U}(x, y)\right\rangle \text { and } \\
D & =h_{1}+h_{2}+h_{3},
\end{aligned}
$$

where $D$ is the mean ocean depth. The other layer thicknesses are similarly computed. We can define the layer densities $\rho_{i}$ and the mean density $\rho_{m}$ based on these layer thicknesses. For example,

$$
\begin{aligned}
\rho_{2} & =\frac{1}{h_{2}}\left\langle\int_{-\bar{h}_{L}(x, y)}^{-\bar{h}_{U}(x, y)} \bar{\rho}(x, y, z) d z\right\rangle \text { and } \\
\rho_{m} & =\frac{1}{D}\left(h_{1} \rho_{1}+h_{2} \rho_{2}+h_{3} \rho_{3}\right) .
\end{aligned}
$$


The other layer densities are similarly computed. We define the mean buoyancy of layer $k$ as $b_{k}=\left(\rho_{0}-\rho_{k}\right) g / \rho_{0}$, where $g$ is the gravitational acceleration and $\rho_{0}$ is the reference density, and thus Eq. (4b) may be rewritten in terms of the layer buoyancies as

$$
D b_{m}=h_{1} b_{1}+h_{2} b_{2}+h_{3} b_{3} .
$$

In terms of the forcing applied to the analytical model, we consider the effect of a zonal wind stress (of characteristic magnitude $\tau$ ) applied over the Southern Ocean. In addition, we apply surface buoyancy forcing via a prescribed buoyancy flux (without distinguishing between heat and salt). The fixed buoyancy flux (rather than surface relaxation) boundary conditions make the model more manageable from a theoretical standpoint because the boundary conditions are independent of the layer buoyancies. We acknowledge that results thus derived are only representative of reality to the extent that boundary conditions on the real ocean can be considered as fixed-flux constraints. Certainly processes such as radiation and freshwater input are largely fixed-flux conditions, but other surface buoyancy fluxes (e.g., oceanatmosphere latent and sensible heat exchange) undeniably lead to relaxation boundary conditions.

Three buoyancy fluxes (units of $\mathrm{m}^{4} \mathrm{~s}^{-3}$ ) are applied in the analytical model: buoyancy removal in the south $q_{S}$ and in the north $q_{N}$ and buoyancy addition over the midocean $q_{H}$. The requirement that the ocean is in a state of thermal equilibrium implies that

$$
q_{H}=q_{S}+q_{N} .
$$

These applied fluxes determine the structure of the layers in the analytical model. As shown in Fig. 1, we assume that the upper-layer interface $\rho_{U}$ outcrops in both the south and north at latitudes $\phi_{S}$ and $\phi_{N}$, respectively. In the Southern Ocean, isopycnal gradients are steepest over the ACC latitudes and thus we assume that $\phi_{S}$ is located within the ACC latitude band. To the south of the ACC the formation sites of AABW are located, from which dense water flows downward to fill the abyssal ocean (layer 3). The formation of North Atlantic Deep Water (NADW) in the far north generates a less dense layer (layer 2) that overlies the AABW (e.g., Sloyan and Rintoul 2001). Thus the abyssal-layer interface $\rho_{L}$ is assumed to outcrop over the ACC in the south at latitude $\phi_{S}$ and shoal on topography in the deep ocean in the north. The choice of the same southern outcrop positions for both layer interfaces is significant, and we discuss this simplification in detail below. We treat the surface outcroppings as instantaneous (steplike) in the analytical model. The freedom in the choice of the layer end latitudes $\phi_{S}$ and $\phi_{N}$ allows us to assume that $q_{S}$ corresponds to a buoyancy loss entirely from layer $3 ; q_{N}$ corresponds to a buoyancy loss entirely from layer 2 ; and, consequently, $q_{H}$ corresponds to a buoyancy addition entirely to the layer 1 . In this way, each layer is subject to a single known external buoyancy source or sink.

Given the above conceptual framework, there are five distinct interlayer transports as shown in Fig. 1: three horizontal flows through the "ends" of the layers and two upwelling fluxes through the bases of the layers. Transports of water from layer $i$ to layer $j$ are denoted as $T_{i j}$, although in some forcing regimes certain flow directions (specifically that of $T_{31}$ ) may be reversed. We can write down two independent equations for volume conservation within each layer,

$$
\begin{aligned}
& T_{12}=T_{31}+T_{21}, \quad \text { layer } 1 \text { and } \\
& T_{23}=T_{32}+T_{31}, \quad \text { layer } 3 .
\end{aligned}
$$

The layer-2 balance (not shown) is simply the sum of the layer- 1 and layer-3 balances.

The next step is to introduce the condition that the net buoyancy of each layer must be constant at equilibrium. With a vertical diffusivity of $\kappa_{v}$, the buoyancy conservation equation at equilibrium may be written as

$$
\iint_{\mathbf{S}_{i}}\left(b \mathbf{u}-\kappa_{v} \frac{\partial b}{\partial z} \hat{\mathbf{z}}\right) \cdot d \mathbf{S}_{i}=0
$$

where $\mathbf{S}_{i}$ is the surface bounding layer $i$ and $\mathbf{z}$ is a unit vertical vector. Thus, we have the possibility of advective buoyancy transfer for all boundaries and also diffusive transfer for horizontally oriented boundaries. The analytical model developed above has instantaneous changes in buoyancy across layer boundaries. This means that a velocity $\mathbf{u}$ across a boundary from layer $k$ to layer $m$ fluxes water of buoyancy $b_{k}$ through the boundary, whereupon it is instantaneously converted (within layer $m)$ to buoyancy $b_{m}$. Thus,

$$
\sum_{i j}\left(b_{k} T_{i j}+q_{i j}\right)=0,
$$

where the sum is over all interfaces $i j$ of layer $j$, and $k$ $(=i$ or $j$ ) is correctly chosen as the layer from which the volume flux is sourced. The factor

$$
q_{i j}=-\kappa_{v}\left\langle\frac{\partial b}{\partial z}\right\rangle_{i j} \hat{\mathbf{z}} \cdot \mathbf{A}_{i j}
$$

is the net diffusive flux of buoyancy out of layer $i$ at interface $i j$, which has area $A_{i j}$, and angled brackets denote horizontal averaging over this area. Applying the 
prescribed surface buoyancy flux boundary conditions (i.e., $q_{H}, q_{N}$, and $q_{S}$ for each of layers 1,2 , and 3 , respectively) to Eq. (9), we can write down two independent buoyancy balances,

$q_{H}+b_{2} T_{21}-q_{12}-b_{1} T_{12}+\left\{\begin{array}{l}b_{3} \\ b_{1}\end{array}\right\} T_{31}=0, \quad$ layer 1

and

$-q_{S}-\left\{\begin{array}{l}b_{3} \\ b_{1}\end{array}\right\} T_{31}+b_{2} T_{23}-b_{3} T_{32}+q_{23}=0, \quad$ layer 3.

The top element in the curly brackets is used when $T_{31}>$ 0 and the bottom element is used when $T_{31}<0$. Note that the buoyancy balance for layer 2 is not independent of the layer- 1 and layer- 3 balances but merely reproduces the zero net flux condition of Eq. (6).

The choice of the same instantaneous southern outcrop position (i.e., $\phi_{S}$ ) for the upper- and lower-layer interfaces is a notable simplification of the above analytical model (and prevents it from being physically representative of actual water mass pathways in this region). We justify this simplification by treating the shaded area in Fig. 1, representing the ACC region, as a zone of intense mixing (or "mixed region") with uniform buoyancy $b_{\text {mix }}$. Winds flux water northward out of this zone and eddies southward into (and through) this zone. The mixed region is subject to a surface buoyancy flux that is some fraction of $q_{S}$ (plus possibly some fraction of $q_{H}$ ), and the remainder of $q_{S}$ is applied to layer 3. Let us consider the adiabatic limit where all diffusive fluxes (e.g., $T_{32}, q_{23}$ ) are zero. In this limit, there are no interlayer fluxes of any sort communicating with layer 3 (see Fig. 1), and thus there exists no capacity to compensate for surface buoyancy loss from layer 3 via $q_{S}$. Therefore, the entirety of $q_{S}$ must be applied to the mixed region. Let us now relax the adiabatic constraint and consider the buoyancy balance pertaining to layer 3 . A water volume of $T_{32}$ is fluxed from the mixed region through layer 3 and ultimately up into layer 2, with a simultaneous diffusive buoyancy flux to layer 3 of $q_{23}$. The corresponding layer-3 buoyancy balance [following Eq. (9)] is thus

$$
b_{\text {mix }} T_{32}-b_{3} T_{32}+q_{23}=0 \Rightarrow b_{\text {mix }}=b_{3}-\frac{q_{23}}{T_{32}}<b_{3} .
$$

However, this statement is inconsistent because it requires that the mixed region, which overlies layer 3 , be less buoyant than layer 3 . Our conclusion is that the mixed region is itself mixed into layer 3 . We therefore amalgamate the mixed region with layer 3 and arrive at the formulation of the model described previously. Note that only two assumptions have been made in this process: (i) a mixed region exists in the south and (ii) the analytical model must be valid in the adiabatic limit. We argue that these assumptions are sensible and thus that the model as formulated is robust.

As observed in section 1, the ACC is closely connected to the MOC and it is desirable that an analytical model of the MOC is also able to describe the ACC transport. The ACC transport scale in the three-layer model may be diagnosed by integrating the thermal wind equation over the ocean depth (with reference to Fig. 1) to yield

$$
T_{\mathrm{ACC}} \sim \frac{1}{2\left|f_{\mathrm{ACC}}\right|}\left(\Delta b_{13} h_{1}^{2}+\Delta b_{23} h_{2}^{2}+2 \Delta b_{23} h_{1} h_{2}\right),
$$

where $\Delta b_{i j} \equiv\left(b_{i}-b_{j}\right)$ and $f_{\mathrm{ACC}}$ is the characteristic value of the Coriolis parameter over the ACC. This derivation makes the assumption that the isopycnal interface $\rho_{L}$ coincides with the streamlines forming the base of the ACC, which does not preclude the nominal (basin averaged) abyssal-layer interface depth (i.e., $z=$ $-h_{1}-h_{2}$ ) exceeding the Drake Passage sill depth.

The full set of six equations describing the three-layer model in terms of the volume fluxes, layer thicknesses, and layer buoyancies is composed of Eqs. (3b), (5), (7), and (11). We now attempt to parameterize (section 2a) the interlayer volume fluxes in terms of the six layer parameters (i.e., $h_{1}, h_{2}, h_{3}, b_{1}, b_{2}$, and $b_{3}$ ) and thereby solve a special case (section $2 b$ ) of the system of equations.

\section{a. Parameterizations}

The presence of the unblocked latitude band of Drake Passage in the south places important restrictions on the transports $T_{31}$ and $T_{23}$, which we assume to be entirely above the Drake Passage sill depth. Following G99 and many other authors, we argue that only wind $\left(T_{\mathrm{Ek}}\right)$ and eddy-driven $\left(T_{e, i}\right)$ meridional transports are possible. Thus, for layer 1 (which includes the Ekman layer) we have that

$$
T_{31}=T_{\mathrm{Ek}}-T_{e, 1}=\frac{\tau L_{x}}{\rho_{0}\left|f_{\mathrm{ACC}}\right|}-T_{e, 1},
$$

where the scale $L_{x}$ is the zonal width of the ocean basin at the latitude of the ACC. For layer 2 (which is below the Ekman layer but above the sill of Drake Passage), we have

$$
T_{23}=T_{e, 2} \text {. }
$$


The eddy transports have the form

$$
T_{e}=\frac{\overline{v^{\prime} b^{\prime}}}{\bar{b}_{z}} L_{x}=L_{x} \kappa_{e} \frac{\bar{b}_{y}}{\bar{b}_{z}},
$$

where we have scaled $\overline{v^{\prime} b^{\prime}}$ similarly to Marshall and Radko (2003) as proportional to the mean meridional buoyancy gradient and an eddy diffusivity $\kappa_{e}$. The diffusivity should not be expected to be constant and will depend on the tendency of the local (ACC) flow to trigger baroclinic instability. Thus, we argue that the eddy diffusivity should scale like

$$
\kappa_{e}=c_{e} \Delta U_{i} L_{S},
$$

where $\Delta U_{i}$ is the change in the zonal velocity over layers $i=1,2$ (i.e., the baroclinic velocity associated with the layer), $L_{S}$ is the meridional length scale of the ACC region, and $c_{e}$ is a universal dimensionless constant [following Visbeck et al. (1997), with $c_{e}$ determined by them to take a value of $0.015 \pm 0.005]$. The required ACC velocity can be calculated from the thermal wind equation as

$$
\Delta U_{i}=\frac{\left(b_{i}-b_{3}\right) h_{i}}{\left|f_{\mathrm{ACC}}\right| L_{S}} .
$$

Thus, the final scaling for the eddy transport (following Visbeck et al. 1997) is

$$
\begin{aligned}
T_{e, i} & =L_{x}\left(c_{e} L_{S} \Delta U_{i}\right) \frac{\partial h}{\partial y} \\
& =\frac{c_{e} L_{x}}{\left|f_{\mathrm{ACC}}\right| L_{S}}\left(b_{i}-b_{3}\right) h_{i}^{2} \equiv a_{e, i} \Delta b_{i 3} h_{i}^{2},
\end{aligned}
$$

where we use $a_{x}$ to summarize the constants relevant to a given transport $T_{x}$. We note that this parameterization is similar to that employed in recent related work such as Samelson $(2009,2011)$.

To scale the northern sinking term $T_{12}$, we use the same parameterization as G99 but with the height scale as the upper depth of maximum overturning $h_{1}$ rather than the pycnocline depth, as verified by de Boer et al. (2010). This scaling assumes that water is transported northward in a boundary current driven by a buoyancy difference between the middle and high latitudes. It has the form

$$
T_{12}=\frac{C}{\beta L_{N}}\left(b_{1}-b_{2}\right) h_{1}^{2} \equiv a_{12} \Delta b_{12} h_{1}^{2},
$$

where $C$ is a constant dependent on the geometry of the boundary current, $\beta$ is the change in the Coriolis parameter with latitude at $\phi_{N}$, and $L_{N}$ is the meridional length scale pertaining to the buoyancy difference.

The explicit scaling of the diffusive fluxes of mass and buoyancy in the ocean interior (i.e., $T_{32}, T_{21}, q_{23}$, and $q_{12}$ ) presents significant difficulties. In two-layer pycnocline models, the argument is simple: the assumption of an exponentially decaying buoyancy distribution and vertical advective-diffusive balance results in a constant upwelling velocity $w=\kappa_{v} / D$, where $D$ is the pycnocline (or buoyancy $e$-folding) depth scale. However, in our three-layer model the vertical velocity will be different at the two layer interfaces; that is, there is no reason to believe that $T_{32}$ and $T_{21}$ are equal. Furthermore, although we expect advective-diffusive balance to hold on a large scale, it need not necessarily be true that it holds at each individual layer interface. Given these complexities, we leave the diffusive fluxes unparameterized and solve the system in the adiabatic limit.

\section{b. The adiabatic limit}

We set $\kappa_{v}=0$ and thus all diffusive fluxes vanish. This vastly simplifies the system volume balance [Eq. (7)] to a single flow pathway of the form

$$
T_{U}=T_{12}=T_{23}=T_{31},
$$

where $T_{U}$ is the upper cell volume transport. This pathway is illustrated in Fig. 1. Note that we must have $T_{31}>0$ for circulation to occur; that is, the Ekman flux must exceed the layer-1 eddy flux, and thus we rely on winds for circulation as might be expected in the adiabatic limit (e.g., Nikurashin and Vallis 2011). A volume flux $T_{U}$ of water is subducted in the North Atlantic and flows south in layer 2 all the way to the ACC latitudes. The water is then fluxed across/into the ACC region (into layer 3 ) by the action of mesoscale eddies, condensed by the action of buoyancy flux $q_{S}$, and then pushed northward by the surface wind stress. The interaction with the abyssal layer (layer 3 ) is a crucial point. The abyssal layer is established by net surface buoyancy removal at rate $-q_{S}$ from the layer (as per Hughes and Griffiths 2006), and thus in order for buoyancy conservation to be satisfied there must be volume fluxes to and from the layer that (in a net sense) add buoyancy at a rate $+q_{S}$. Hence, $T_{31}$ and $T_{23}$ must communicate with layer 3 (as formulated above) for any nonzero $q_{S}$. The communication will be constrained by the thermodynamic balances for the layers.

The buoyancy balances relevant to the adiabatic system are readily derived from Eq. (11) as 


$$
\begin{aligned}
& q_{H}=\Delta b_{13} T_{U} \quad \text { and } \\
& q_{S}=\Delta b_{23} T_{U} .
\end{aligned}
$$

Substituting the transport parameterizations into the volume balance [Eq. (21)] yields

$$
T_{\mathrm{Ek}}-a_{e, 1} \Delta b_{13} h_{1}^{2}=a_{12} \Delta b_{12} h_{1}^{2}=a_{e, 2} \Delta b_{23} h_{2}^{2} .
$$

We thus have four independent equations [Eqs. (22) and (23)] on four variables $\left(h_{1}, h_{2}, \Delta b_{12}, \Delta b_{23}\right)$, which may be solved analytically to yield

$$
\begin{aligned}
h_{1} & =\left(\frac{T_{\mathrm{Ek}} \sqrt{q_{N}}}{q_{H}}\right) \frac{\gamma}{\sqrt{a_{12}}}, \\
h_{2} & =h_{1}\left(\frac{a_{12} q_{N}}{a_{e, 2} q_{S}}\right)^{1 / 2}=\left(\frac{T_{\mathrm{Ek}} q_{N}}{q_{H} \sqrt{q_{S}}}\right) \frac{\gamma}{\sqrt{a_{e, 2}}}, \\
\Delta b_{12} & =\left(\frac{q_{H}}{T_{\mathrm{Ek}}}\right) \frac{1}{\gamma} \\
\Delta b_{23} & =\left(\frac{q_{S}}{q_{N}}\right) \Delta b_{12}=\left(\frac{q_{H} q_{S}}{T_{\mathrm{Ek}} q_{N}}\right) \frac{1}{\gamma}, \text { and } \\
\Rightarrow \Delta b_{13} & =\Delta b_{12}+\Delta b_{23}=\left(\frac{q_{H}^{2}}{q_{N} T_{\mathrm{Ek}}}\right) \frac{1}{\gamma},
\end{aligned}
$$

where $\gamma$ is a dimensionless number defined as

$$
\gamma=\left(\frac{q_{N}}{q_{H}}+\frac{a_{e, 1}}{a_{12}}\right)^{-1} .
$$

The number $\gamma$ is closely related to the degree of eddy compensation occurring in layer 1 and transport $T_{31}$. The fractional eddy compensation in the surface layer is

$$
\mathcal{C}=\frac{T_{e, 1}}{T_{\mathrm{Ek}}}=\gamma \frac{a_{e, 1}}{a_{12}}=\frac{\frac{a_{e, 1}}{a_{12}}}{\frac{q_{N}}{q_{H}}+\frac{a_{e, 1}}{a_{12}}} .
$$

We make the assumption that eddies are significant, implying that $\mathcal{C}$ is on the order of 1 (but obviously not equal to 1 , because then there would not be any circulation) and thus $\gamma \sim a_{12} / a_{e, 1}$. We will use this approximation for $\gamma$ henceforth because it significantly simplifies the scales of Eq. (24).

Returning to Eq. (24), we note that both layer thicknesses 1 and 2 are increased by the action of winds (due to winds removing water from layer 3) and both buoyancy differences are reduced by the action of winds (due to winds causing stirring). Another key feature of the equations is that, despite the stagnant nature of the abyssal layer in the adiabatic limit, the buoyancy flux $q_{s}$ is still vitally important in determining the ocean stratification (as described by the layer thicknesses and buoyancies) and therefore exerts control on the circulation. The occurrence of $q_{S}$ in the equations is also logical. For example, if $q_{S} \rightarrow 0$ then Eq. (24) predicts that layer 2 will become indistinguishable from layer 3 $\left(\Delta b_{23} \rightarrow 0\right)$ and that the size of layer 3 will shrink or equivalently that layer 2 will expand to fill the entire deep ocean $\left(h_{2} \rightarrow \infty\right)$.

The scale for the North Atlantic overturning (and upper cell transport) can be derived from Eqs. (24a) and $(24 c)$ as

$$
T_{U}=T_{12} \sim \Delta b_{12} h_{1}^{2} \sim \frac{q_{N} \tau}{q_{H}} .
$$

This dependence on the forcing parameters is readily explained. First, larger wind stress $\tau$ increases the Ekman volume flux into layer 1 in the Southern Ocean and thus increases the overturning magnitude, despite partial compensation by the southward eddy flux. This prediction is consistent with G99 and indeed with many other models, including the adiabatic scaling theory of Wolfe and Cessi (2011). However, none of these theories predict the buoyancy forcing dependence described by Eq. (27). An increased northern buoyancy loss $q_{N}$ enables an increased conversion of water with buoyancy $b_{1}$ to water with buoyancy $b_{2}$ in the north and thus increases the overturning magnitude. The action of buoyancy addition $q_{H}$ to layer 1 is to increase the buoyancy $b_{1}$ and thus the buoyancy difference $\Delta b_{12}$. Consequently, for a given $q_{N}$, a lesser amount of water is able to be converted to buoyancy $b_{2}$, thus reducing the overturning magnitude. We note that the scale for $T_{12}$ may also be written as

$$
T_{12} \sim \tau\left(1-\frac{q_{S}}{q_{H}}\right)=\frac{\tau}{1+\frac{q_{S}}{q_{N}}},
$$

using the zero net flux condition [Eq. (6)], and thus it is incorrect to say that the North Atlantic overturning is independent of $q_{S}$. Any change in $q_{S}$ must involve a compensatory change in either $q_{N}$ or $q_{H}$ (or both) if we are to have the ocean in a state of thermodynamic equilibrium. However, we can state that the buoyancy forcing dependence is completely described by the ratio of the southern to the northern forcing $q_{S} / q_{N}$. If this ratio is small, then the overturning is insensitive to changes in buoyancy forcing and varies according to the wind stress. 
We have not yet considered scales for the ACC transport and abyssal overturning because the abyssal overturning is zero in the adiabatic limit and the ACC transport is closely linked to the abyssal overturning (see section 4). Scales for both of these quantities will be derived in section 4 .

The adiabatic limit solutions [Eq. (24)] can be applied to derive scales for the buoyancies (with respect to fixed mean buoyancy $b_{m}$ ) of the three layers in the analytical model. The equilibrium buoyancy of the surface layer can be written as

$b_{1}-b_{m}=\left(b_{3}-b_{m}\right)+\Delta b_{13} \approx \Delta b_{13} \sim \frac{q_{H}^{2}}{q_{N} \tau}$,

where we make the assumption that (i) $\left|b_{1}-b_{2}\right| \gg$ $\left|b_{3}-b_{m}\right|$ because buoyancy gradients in the upper ocean are far larger than those in the abyssal ocean. The equilibrium buoyancy of the abyssal layer can be derived from Eq. (5) as

$$
\begin{aligned}
b_{3}-b_{m} & =-\frac{1}{D}\left(h_{1} \Delta b_{13}+h_{2} \Delta b_{23}\right) \\
& =-\frac{\sqrt{q_{S}}}{D \sqrt{a_{12}}}\left(\frac{q_{H}}{\sqrt{q_{S} q_{N}}}+\sqrt{\frac{a_{12}}{a_{e, 2}}}\right) \sim-\sqrt{q_{S}} .
\end{aligned}
$$

The final simplification results from assuming that (ii) $h_{2} \gg h_{1} \sim h_{3}$ and applying Eq. (24b) to give the result $a_{12} / a_{e, 2} \gg q_{S} / q_{N} \sim q_{H} / \sqrt{q_{S} q_{N}}$, as long as $q_{S}$ is of similar order to $q_{N}$. Finally, the buoyancy of the middepth layer can be computed by rearranging Eq. (5) as

$$
h_{1}\left(b_{1}-b_{m}\right)+h_{2}\left(b_{2}-b_{m}\right)+h_{3}\left(b_{3}-b_{m}\right)=0
$$

and neglecting the third term, consistent with our previous assumptions (i and ii). Thus,

$$
b_{2}-b_{m} \approx-\frac{h_{1}}{h_{2}}\left(b_{1}-b_{m}\right) \sim-\frac{q_{H}^{2}}{\tau q_{N}} \sqrt{\frac{q_{S}}{q_{N}}} .
$$

We note that the buoyancy of the abyssal layer $b_{3}$ is unique in that it is the only parameter in the entire system that is independent of wind stress. Indeed, buoyancy $b_{3}$ is almost completely determined by the buoyancy flux $q_{S}$. This is unsurprising given that $q_{S}$ is responsible for the formation of layer 3 (which is motionless at adiabatic equilibrium) and no buoyancy can diffuse downward into this layer from the surface flux $q_{H}$ under adiabatic conditions. However, $q_{H}$ strongly controls the upper-ocean buoyancies $b_{1}$ and $b_{2}$ as shown by Eqs. (29) and (32), respectively. These two equations also demonstrate that winds act to stir the upper ocean: the effect of increased winds is to increase $b_{2}$ toward the mean buoyancy and reduce $b_{1}$ toward the mean buoyancy.

We now test the veracity of the above theory with a numerical model. In particular, we investigate to what extent the results derived in the adiabatic limit may be used to describe the generalized diabatic system.

\section{Configuration of the numerical model}

We use the Massachusetts Institute of Technology General Circulation Model (MITgcm; Marshall et al. 1997) in hydrostatic mode with a nominal resolution of $1 / 4^{\circ}$ (at the equator) on a Mercator grid. There are 36 vertical levels over an ocean depth of $4000 \mathrm{~m}$, varying from $10 \mathrm{~m}$ at the surface to $200 \mathrm{~m}$ in the abyssal ocean. Analogous to the above analytical model, the numerical model domain consists of a flat-bottomed single basin of $40^{\circ}$ zonal width extending from approximately $70^{\circ} \mathrm{S}$ to $70^{\circ} \mathrm{N}$ and thus representative of the Atlantic basin. All boundaries are sloped (rather than vertical) and there are continental shelves at $800-\mathrm{m}$ depth and of $3^{\circ}$ meridional width in the north and south. There is a zonally reconnecting channel simulating Drake Passage centered at $59^{\circ} \mathrm{S}$ and of $1800-\mathrm{m}$ depth. The model topography is shown in Fig. 2a. We assume free-slip sidewalls and a no-slip bottom with a quadratic drag of coefficient $C_{D}=0.003$. Increased mixing in the near-surface region is parameterized by a Gaussian vertical diffusivity profile with a decay depth of $150 \mathrm{~m}$ and surface diffusivity of $2 \times 10^{-3} \mathrm{~m}^{2} \mathrm{~s}^{-1}$. The interior vertical diffusivity (and viscosity) is set as $1 \times 10^{-5} \mathrm{~m}^{2} \mathrm{~s}^{-1}$, and biharmonic diffusivity (and viscosity) of $2 \times 10^{11} \mathrm{~m}^{4} \mathrm{~s}^{-1}$ is chosen in the horizontal. We apply a high-order advection scheme (MITgcm scheme 7) known as the seventh-order one-step method with monotonicity-preserving limiter (OS7MP) to minimize numerical diffusion (see Ilicak et al. 2012).

The numerical model is forced identically to the analytical model, via a combination of surface winds and prescribed buoyancy fluxes, both zonally uniform. We only consider the net buoyancy field and net buoyancy fluxes; temperature and salinity are not considered individually. The buoyancy forcing profile is shown in Fig. $2 \mathrm{~b}$. The buoyancy fluxes remove buoyancy north of $\phi_{N}=$ $60^{\circ}$, with magnitude $q_{N}$, and south of $\phi_{S}=-60^{\circ}$, with magnitude $q_{S}$, and add buoyancy over the midocean between $\phi_{S}$ and $\phi_{N}$, with magnitude $q_{H}$ (all in units of $\mathrm{m}^{4} \mathrm{~s}^{-3}$ ). We choose a reference case with a northern buoyancy flux 3 times the magnitude of the southern buoyancy flux (i.e., $q_{N}^{\text {ref }}=3 q_{S}^{\text {ref }}$ ) and a net midocean buoyancy input of $q_{H}^{\text {ref }}=2 \times 10^{5} \mathrm{~m}^{4} \mathrm{~s}^{-3}$. Other runs are then specified relative to the reference case by the triple 

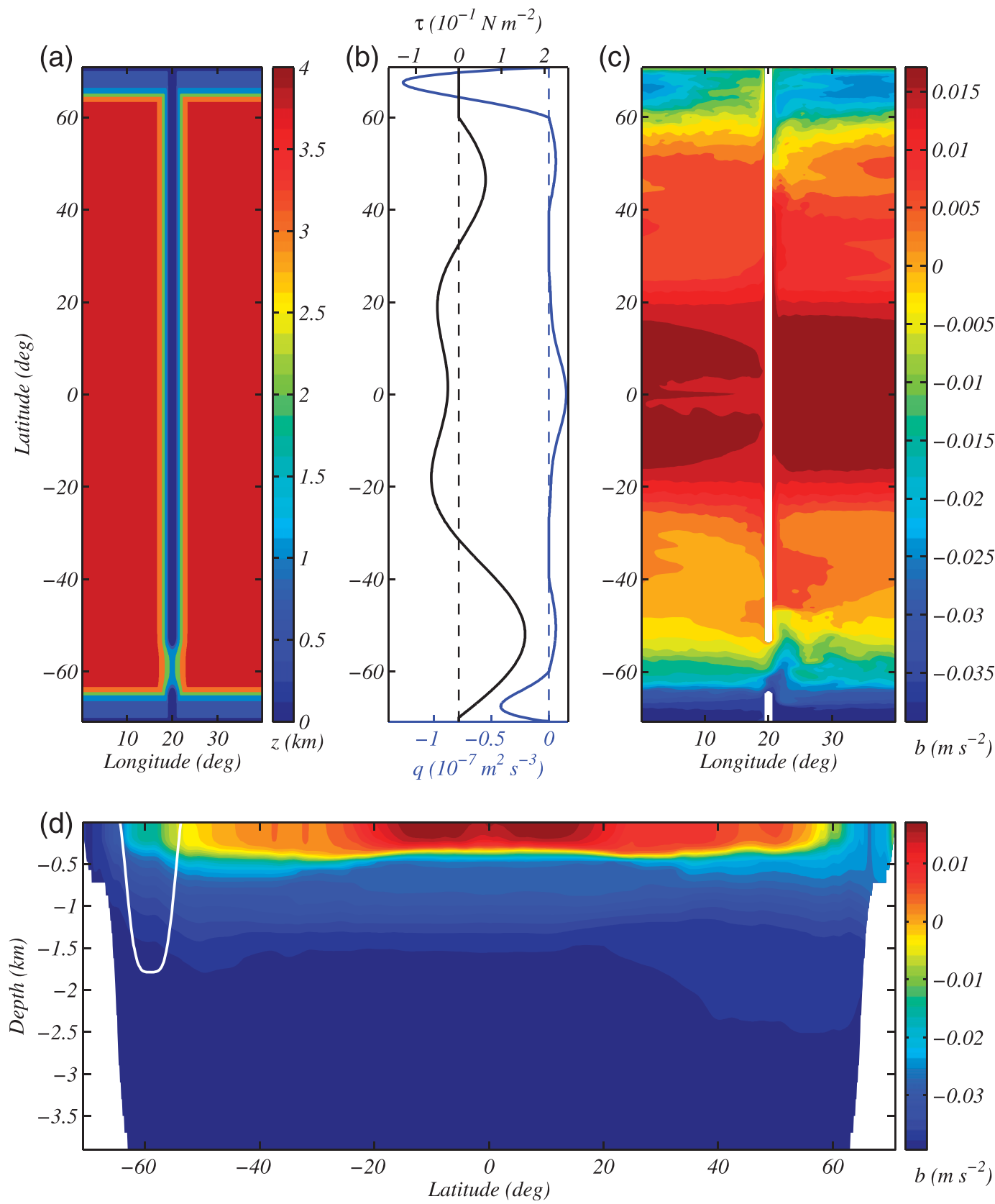

FIG. 2. The configuration of the numerical model. (a) The ocean depth over the model domain. (b) The forcing applied at the surface for the reference case: the wind stress $\tau^{\text {ref }}(y)$ (black) and the buoyancy flux $q^{\text {ref }}(y)$ (blue). (c) The steady-state mean surface buoyancy field $b$ for the reference case. (d) A transect of the steady-state mean buoyancy field through the center of the ocean basin for the reference case. The outline of Drake Passage is marked in white.

$\left(q_{S}^{\dagger}, q_{H}^{\dagger}, \tau^{\dagger}\right)$, where the superscript $\dagger$ implies a quantity scaled with respect to the reference value (e.g., $q_{S}^{\dagger}=q_{S}\left(q_{S}^{\text {ref }}\right)$. The parameter $\tau^{\dagger}$ is the scaling applied to the reference wind profile in Fig. $2 b$ for a given run: that is, $\tau(y)=\tau^{\dagger} \tau^{\text {ref }}(y)$. The relative northern buoyancy flux $q_{N}^{\dagger}$ can be expressed in terms of the other relative buoyancy flux parameters [using Eq. (6)] as $q_{N}^{\dagger}=\left(4 q_{H}^{\dagger}-q_{S}^{\dagger}\right) / 3$.
The above configuration of surface forcing leads to the equilibrium structure shown in Figs. 2c,d. This structure is representative of the real ocean with a buoyant surface layer separated from the dense abyssal ocean by a region of large buoyancy gradients. The densest water (AABW) forms to the south of Drake Passage despite the largest negative buoyancy fluxes being located in the 

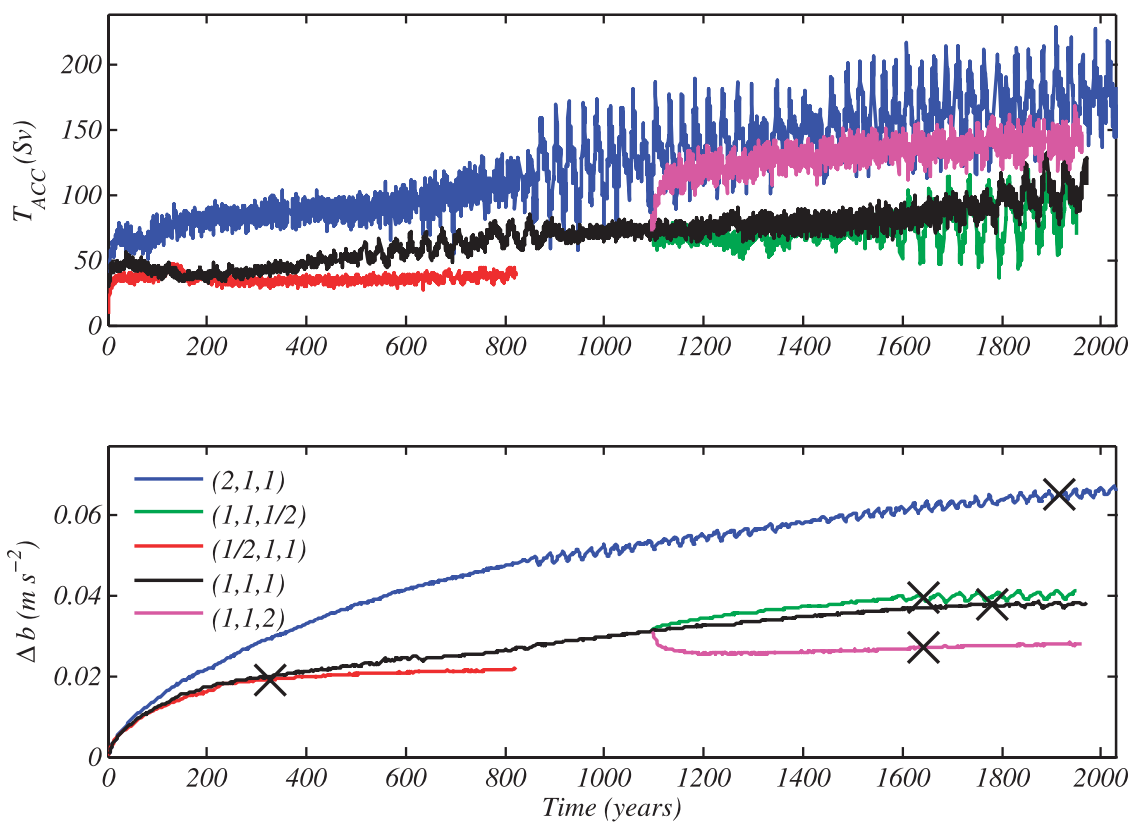

FIG. 3. Equilibration of the numerical model, starting from an initial uniform density state at time zero. The upper graphic shows the evolution of the ACC transport $T_{\mathrm{ACC}}$ with time. The lower graphic shows the evolution of the horizontally averaged top-bottom buoyancy difference $\Delta b=\left\langle b_{\text {top }}-b_{\text {bottom }}\right\rangle$ in time. The reference run is $\left(q_{S}^{\dagger}, q_{H}^{\dagger}, \tau^{\dagger}\right)=(1,1,1)$. Run $(1 / 2,1,1)$ has half and $(2,1,1)$ has twice the reference southern buoyancy forcing with unchanged $q_{H}$ and wind stress. The larger $q_{S}$ increases the equilibration time for the run, as represented by the crosses on each curve, which record when the rate of change of $\Delta b$ with time drops (and remains) below $1.5 \times 10^{-5} \mathrm{~m} \mathrm{~s}^{-2} \mathrm{yr}^{-1}$. The wind stress in the reference run is altered at approximately $1100 \mathrm{yr}$ (with no change to the buoyancy forcing) to give the half-wind $(1,1,1 / 2)$ and double-wind $(1,1,2)$ runs.

north (for the reference case). As can be inferred from the large buoyancy gradients in Fig. 2c, the model possesses a realistic circumpolar current (ACC) with its transport maximum near the southern edge of Drake Passage.

The spinup of the numerical model toward the equilibrium state is shown in Fig. 3, starting from a uniform density state at time zero. We represent the spinup in terms of the ACC transport $T_{\mathrm{ACC}}$ and the horizontally averaged buoyancy difference between the top and bottom layers $\Delta b$, which describes the development of the stratification and MOC. We use the less volatile $\Delta b$ to judge model equilibration. Noting the persistent low-frequency fluctuations in $\Delta b$ and its long timescale asymptotic behavior, we choose the equilibration criterion to be when the 300-yr moving average of the time rate of change of $\Delta b$ drops (and remains) below $1.5 \times$ $10^{-5} \mathrm{~m} \mathrm{~s}^{-2} \mathrm{yr}^{-1}$. Importantly, if this threshold is increased then the qualitative aspects of the results discussed below do not change (although the exact numbers and fit correlations do change; see section 4e). We thus consider the results to be robust.

With reference to Fig. 3, we observe that significant oscillations occur in $\Delta b$ and $T_{\mathrm{ACC}}$ for model runs with small $q_{N}$ (consistent with the damping action of $q_{N}$ observed by Allison et al. 2011) and small wind stress. These oscillations and the details of the equilibration process are the subject of ongoing investigations and will not be addressed in this paper.

\section{Numerical model results}

\section{a. Three-layer structure}

The equilibrium state observed in the numerical model shows a structure that is strongly dependent on the pattern of buoyancy forcing applied at the surface. Each region of buoyancy forcing of a given sign (i.e., $q_{S}, q_{N}$, and $q_{H}$ ) is responsible for the formation and maintenance of a layer, as demonstrated by the structure of the isopycnal-averaged overturning streamfunction plots in Fig. 4. The dashed lines in the figure show the two IOEs that subdivide the ocean depth into counterflowing layers. For model runs with nonzero values of all three surface fluxes, there is a persistent three-layer structure, even when $q_{S} \ll q_{H}$ as for run $(1 / 2,1,1)$ (Fig. $4 \mathrm{~b}$ ). Dense water flows north in the bottom layer and light 

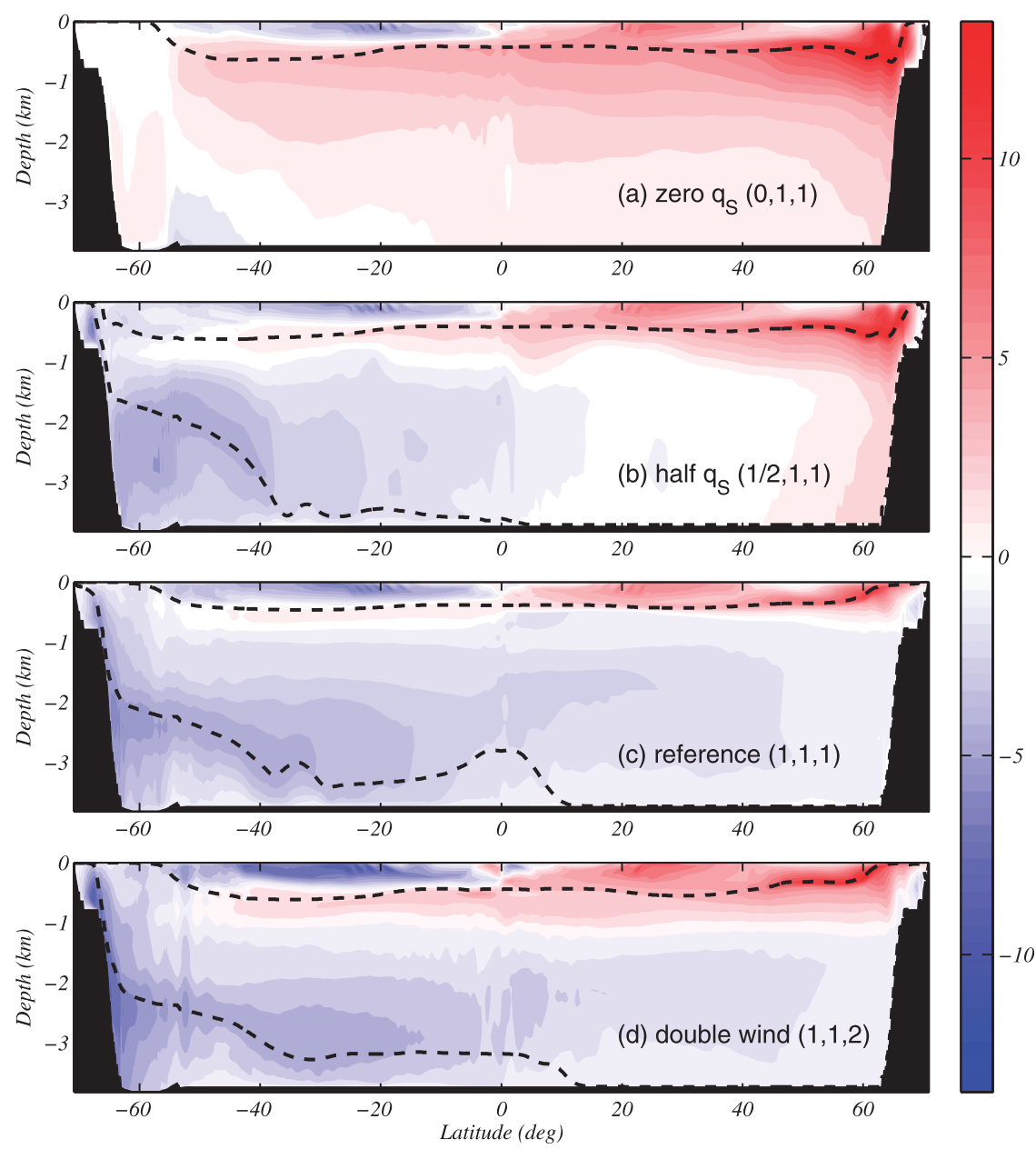

FIG. 4. The meridional overturning streamfunction in Sverdrups for four of the model runs labeled by the triple $\left(q_{S}^{\dagger}, q_{H}^{\dagger}, \tau^{\dagger}\right)$. The plots correspond to the (a) zero southern buoyancy forcing, (b) half southern buoyancy forcing, (c) reference, and (d) double-wind runs. The streamfunction is calculated in density space. Red shading (positive) denotes clockwise circulation, and blue (negative) denotes anticlockwise. The dashed black lines show the IOEs for each of the North Atlantic and abyssal cells that form the layer boundaries in our analytical model. We also note that the IOEs approximately describe lines (in density-averaged latitudedepth space) along which there is no meridional motion, and there are extrema/inflections in the variation of the vertical velocity with depth.

water flows north in the surface layer, with a return southward flow of water (from both overturning cells) in the middepth layer, precisely as formulated in the analytical model. In contrast, setting flux $q_{S}$ to zero [i.e., run $(0,1,1)$; Fig. $4 a]$ means that there is no generation of an abyssal layer and thus much lower abyssal density and no abyssal overturning cell. The North Atlantic overturning cell expands to fill the entire ocean depth at almost all latitudes, and thus the model ocean in run $(0,1,1)$ possesses a fundamentally different (two layer) structure. This difference emphasizes the vital importance of flux $q_{S}$ in generating a realistic stratification and circulation.
We now compare the impacts of wind and buoyancy forcing on the overturning streamfunctions. The outcrop positions of the IOEs are determined almost exclusively by the relative magnitudes of the three surface buoyancy flux parameters. In particular, the dominance of the abyssal cell/layer, as measured by its northward penetration, is a strong function of $q_{s}$. Referring to Fig. 4, a reduced value of $q_{S}$ [e.g., $(1 / 2,1,1)$; Fig. $\left.4 \mathrm{~b}\right]$ results in a less dominant abyssal cell that is unable to penetrate significantly into the Northern Hemisphere, as well as a decreased abyssal overturning magnitude. In contrast, an increased wind strength [e.g., $(1,1,2)$; Fig. 4d] does not significantly alter the lower IOE outcrop position 

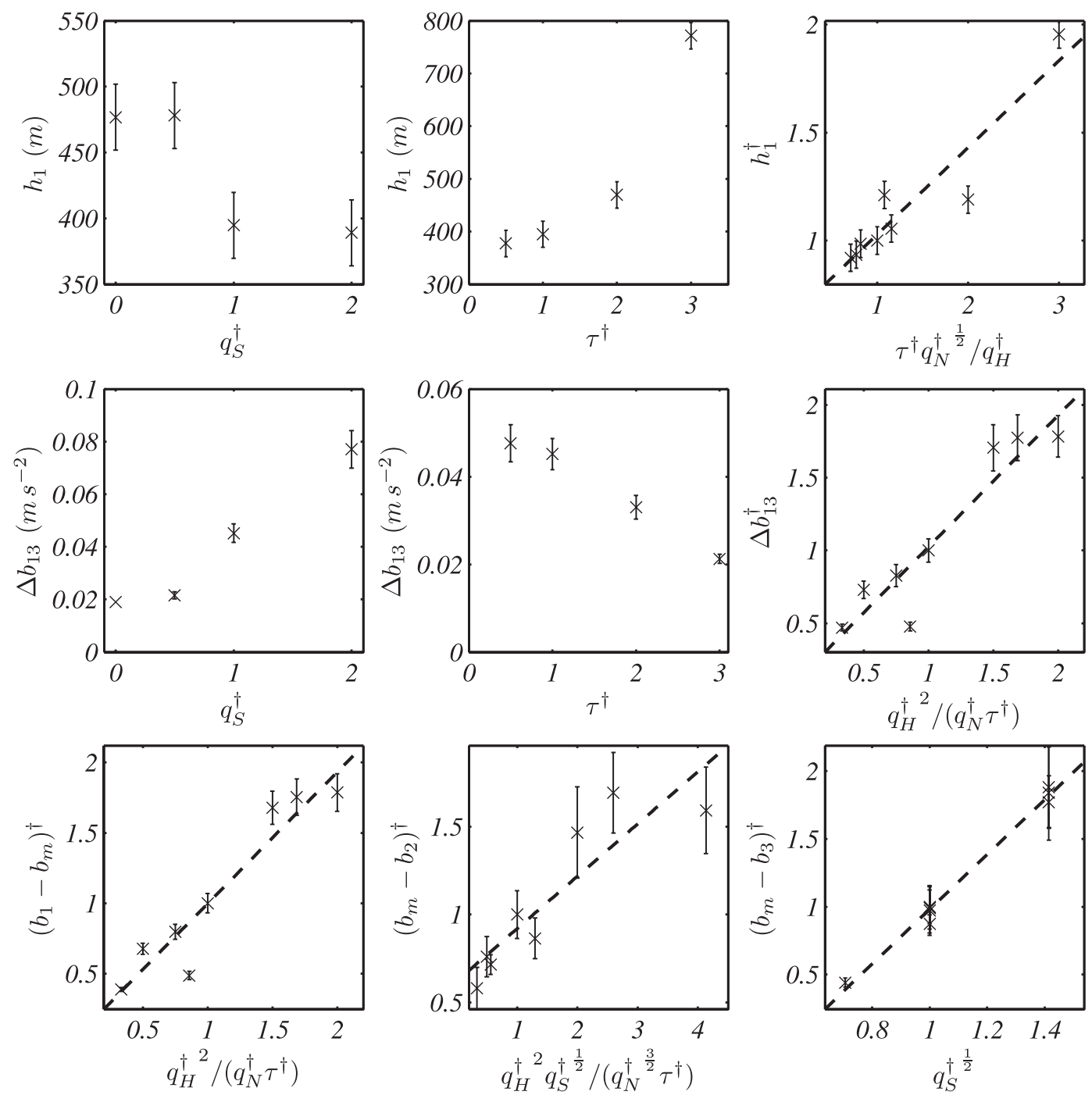

FIG. 5. The response of the ocean stratification to changing surface forcing. (top) The response of the layer-1 thickness to changing (left) $q_{S}^{\dagger}$ and (middle) $\tau^{\dagger}$, for reference values of the other independent forcing parameters, and (right) in terms of the adiabatic limit scaling. (middle) As in (top), but for the full-depth buoyancy difference. (bottom) The variation of the (left) layer-1, (middle) layer-2, and (right) layer-3 buoyancies (with respect to the mean buoyancy $b_{m}$ ) in terms of the appropriate adiabatic limit scale. Plots using derived scales do not show runs with zero $q_{S}$ (because there are only two layers and thus three-layer scales are not applicable) or those with $\tau^{\dagger}<1$ (because the Ekman transport is insufficient for the adiabatic limit to be a valid approximation). The error bars display the estimated uncertainties, computed as follows: $\delta h_{1}=\delta h_{U}, \delta b_{1}=\left(b_{1}-b_{2}\right) \delta h_{U} / h_{1}, \delta b_{3}=\left(b_{2}-b_{3}\right) \delta h_{L} / h_{3}, \delta b_{2}=\left(b_{1}-b_{2}\right) \delta h_{U} / h_{2}+$ $\left(b_{2}-b_{3}\right) \delta h_{L} / h_{2}$, and $\delta\left(\Delta b_{i j}\right)=\delta b_{i}+\delta b_{j}$.

or northward penetration of the abyssal cell but does significantly decrease the strength of the abyssal overturning. Increased winds also significantly increase the surface-layer depth and the strength of the North Atlantic overturning. However, unlike the increased northern buoyancy flux $q_{N}$ in run $(1 / 2,1,1)$, increased winds do not expand the North Atlantic cell to full depth. Thus, each layer is strongly controlled by its corresponding surface buoyancy flux, with winds then modifying the layer depths and overturning magnitudes.
The response of the ocean stratification to changing wind and buoyancy forcing is shown in Fig. 5 in terms of the analytical model buoyancy and height scales. The action of buoyancy flux $q_{S}$ is (i) to decrease the surfacelayer thickness $h_{1}$ and (ii) to increase the full-depth buoyancy difference $\Delta b_{13}$. Both effects are readily explained. A greater $q_{S}$ means that more water of a given buoyancy $b_{1}$ from layer 1 is able to be converted to a given buoyancy $b_{3}$ and thus the flux of water out of layer 1 increases, reducing the volume and thickness 

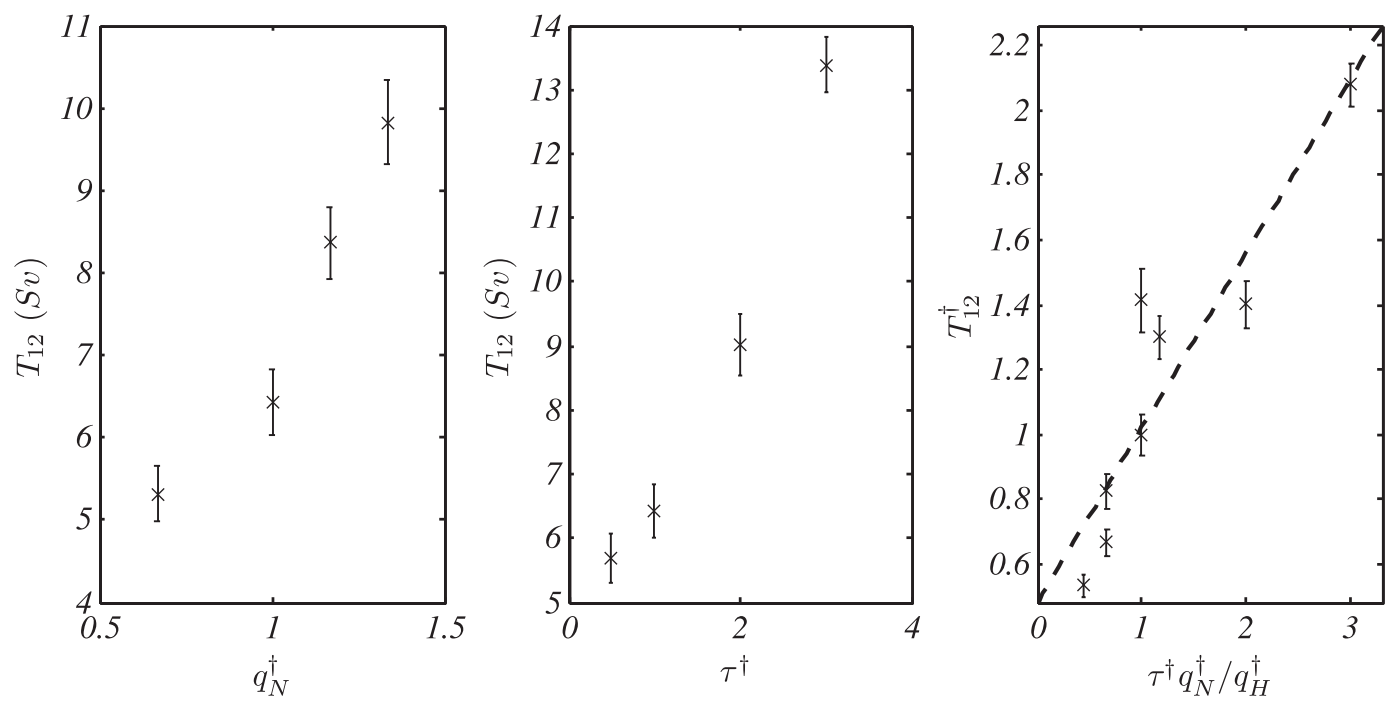

FIG. 6. The response of the North Atlantic overturning $T_{12}$ to (left) changing northern buoyancy flux and (middle) wind stress, for reference values of the other independent forcing parameters. (right) The variation of the overturning with the adiabatic limit scale is also shown. Uncertainty in the value of $T_{12}$ for each run is estimated as $\delta T_{12} \approx \delta h_{U} T_{12} /$ $h_{1}$, where $2 T_{12}$ is the typical change in $\psi$ over a height scale for the North Atlantic cell of $\sim 2 h_{1}$ (see Fig. 4).

of the layer. Conversely, for a given flux of water out of layer 1 , greater $q_{S}$ allows water of buoyancy $b_{1}$ to be condensed to a lower buoyancy $b_{3}$ and thus $\Delta b_{13}$ increases. The surface wind stress also has strong control over the layer thicknesses and buoyancy differences. Increased wind stress leads to a greater Ekman transport from layer 3 into layer 1 in the Southern Ocean, thus increasing the volume and thickness of layer 1 but simultaneously reducing the buoyancy of layer 1 due to the mixing in of the dense water from layer 3 . Thus, the buoyancy difference $\Delta b_{13}$ is reduced with increasing wind stress. The other buoyancy differences (i.e., $\Delta b_{12}$ and $\Delta b_{23}$ ) vary in the same manner (though with different magnitudes) with the wind stress and buoyancy forcing.

Figure 5 also displays the adiabatic limit scaling for each of the layer buoyancies, layer-1 thickness, and fulldepth buoyancy difference, as derived in section 2 . The adiabatic scales displayed are broadly consistent with the forcing response of the numerical model, accounting for typical uncertainties (indicated by the error bars). Uncertainties in the interface heights $\left(h_{U}\right.$ and $\left.h_{L}\right)$ are approximately half the vertical grid spacing at those depths in the numerical model; that is, $\delta h_{U}=25 \mathrm{~m}$ and $\delta h_{L}=100 \mathrm{~m}$ for the upper and lower interfaces, respectively. The uncertainty induced in $b_{1}$, for example, can then be estimated as $\delta b_{1} \approx\left(b_{1}-b_{2}\right) \delta h_{U} / h_{1}$ and similarly for the other layer buoyancies (as detailed in the figure captions). The relatively large uncertainty in the lower interface height causes uncertainties in the values of the layer-2 and layer-3 thicknesses that are comparable with the forcing responses of these parameters, and thus the accuracy (or otherwise) of the corresponding adiabatic scales cannot be determined.

Diabatic effects result in a number of deviations from the adiabatic predictions. First, in some of the scaling plots of Fig. 5 there is a nonzero $y$ intercept (e.g., for $h_{1}$ ) presumably resulting from the action of diffusion. Also, the buoyancy differences $\Delta b_{12}$ and $\Delta b_{23}$ (not shown in the figure) do not scale with the adiabatic predictions. This is unsurprising given that these two quantities correspond to the buoyancy differences across the interior-layer interfaces where diffusive fluxes (i.e., $T_{32}$, $T_{21}, q_{23}$, and $q_{12}$ ) will be present. Thus, the values of the buoyancy differences will be modified according to the advective-diffusive balance in the ocean interior and thus scale differently to the adiabatic case. Despite these differences and given the veracity of the adiabatic scales for the individual layer buoyancies, full-depth buoyancy difference $\Delta b_{13}$, and surface-layer thickness, it appears that the numerical model is close to the adiabatic limit.

\section{b. North Atlantic overturning}

The response of the North Atlantic overturning to changing northern buoyancy forcing and wind stress is plotted in Fig. 6. The overturning $T_{12}$ generally increases with both $q_{N}$ and $\tau$, with some variation due to the $q_{H}$. All three controls are included in the adiabatic limit scale $T_{12} \sim \tau q_{N} / q_{H}$ [see Eq. (27)], also shown in the figure. Once again the adiabatic scaling functions reasonably well in describing the numerical model results. 

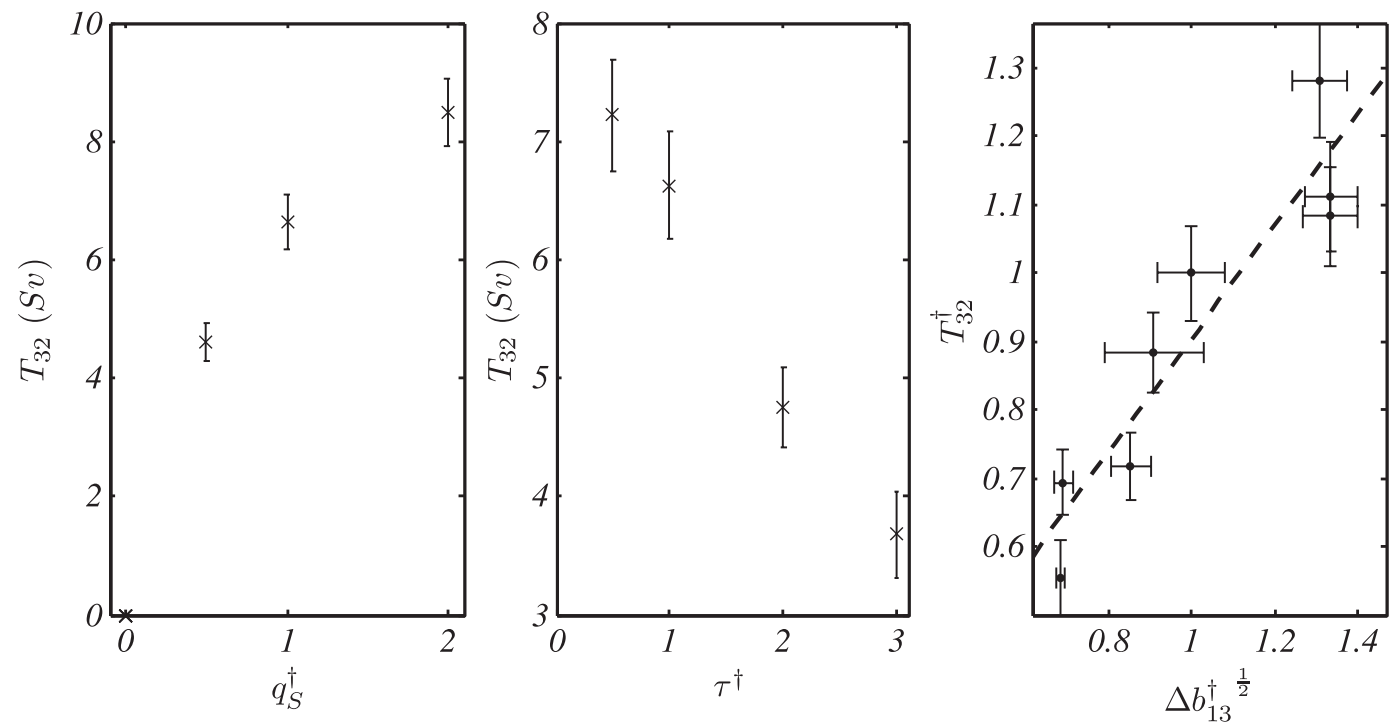

FIG. 7. The response of the abyssal overturning $T_{32}$ to (left) changing southern buoyancy flux and (middle) wind stress, for reference values of the other independent forcing parameters. (right) The variation of the overturning with the adiabatic limit scale is also shown. The abyssal overturning oscillates similarly to the ACC transport in runs with large $q_{S}$ (as described in section 3), making it difficult to obtain an accurate value for $T_{32}$. However, in some time intervals the oscillations are damped and the values of $T_{32}$ presented here for the strongly oscillating runs (i.e., $q_{S}^{\dagger}=2$ and $\tau^{\dagger}=1 / 2$ ) are extracted over these intervals. Uncertainty in the value of $T_{32}$ for each run is estimated as $\delta T_{32} \approx$ $2 \delta h_{L} T_{32} / h_{2}$, where $2 T_{32}$ is the typical change in $y$ over a height scale for the abyssal cell of $\sim h_{2}$ (see Fig. 4). The uncertainty in $\Delta b_{13}$ is computed as $\delta\left(\Delta b_{13}\right)=\left(b_{1}-b_{2}\right) \delta h_{U} / h_{2}+\left(b_{2}-b_{3}\right) \delta h_{L} / h_{3}$, as described previously.

Departures from the scaling are presumably due to nonnegligible diffusive effects and also changes in the geometry of the boundary current [i.e., the value of $C$ in Eq. (20)] when the North Atlantic overturning cell expands to the full-ocean depth, as in run $(1 / 2,1,1)$.

\section{c. Abyssal overturning}

The abyssal overturning tends to increase with southern buoyancy forcing and decrease with wind stress, as illustrated in Fig. 7. The behavior with wind stress is unsurprising given that increased Ekman transport tends to push more water out of the abyssal layer (and the abyssal MOC cell) and into the surface layer (and the North Atlantic MOC cell). Thus, as the North Atlantic overturning $T_{12}$ increases with wind stress, the abyssal overturning $T_{32}$ must decrease.

The effect of the southern buoyancy forcing on the abyssal overturning is most easily explained by observing that the diffusive upwelling $T_{32}$ in the midlatitudes is sourced from the flux of water flowing into layer 3 in the south. Referring to the overturning plots of Fig. 4, the abyssal IOE runs parallel and very close to the southern continental slope, implying that the flux of water into layer 3 is in the form of a thin, intense gravity current down the slope. A greater flux $q_{S}$ leads to denser water at the surface, which flows at a greater speed down the slope, and so the volume flux $T_{32}$ increases with $q_{S}$. To make this clearer, let us consider the momentum balance for the gravity current. As per Killworth (2001), we take the dynamical balance to be between the downslope buoyant acceleration and a quadratic drag of coefficient $C_{D}$. The current velocity then scales like $U^{2} \sim g^{\prime} D / C_{D}$, where $D$ is an appropriate distance scale and $g^{\prime}$ is an appropriate reduced gravity. As the current flows over nearly the entire ocean depth, we take $D$ to be constant and $g^{\prime}$ to be equal to the full-depth buoyancy difference $\Delta b_{13}$. Assuming the size/shape of the current are fixed, we have a scale for the abyssal overturning of $T_{32} \sim \sqrt{\Delta b_{13}} \sim q_{H} / \sqrt{\tau q_{N}}$, where we have applied the adiabatic scale for $\Delta b_{13}$ [Eq. (24e)]. As shown in Fig. 7, this scale describes the forcing response of the abyssal overturning in the numerical model (to within estimated uncertainties). Nikurashin and Vallis (2011) predict a similar inverse dependence on wind stress in the small diffusion limit of their theoretical model.

\section{d. ACC transport}

The ACC transport increases with both the southern buoyancy forcing $q_{S}$ and the wind stress $\tau$, as shown in Fig. 8. We will use the three-layer ACC transport scale [Eq. (13)] to describe this observed forcing response. First, because $h_{2} \gg h_{1}$, the third term of Eq. (13) may be neglected as small compared with the second term. We can then resolve the remaining terms into MOC 

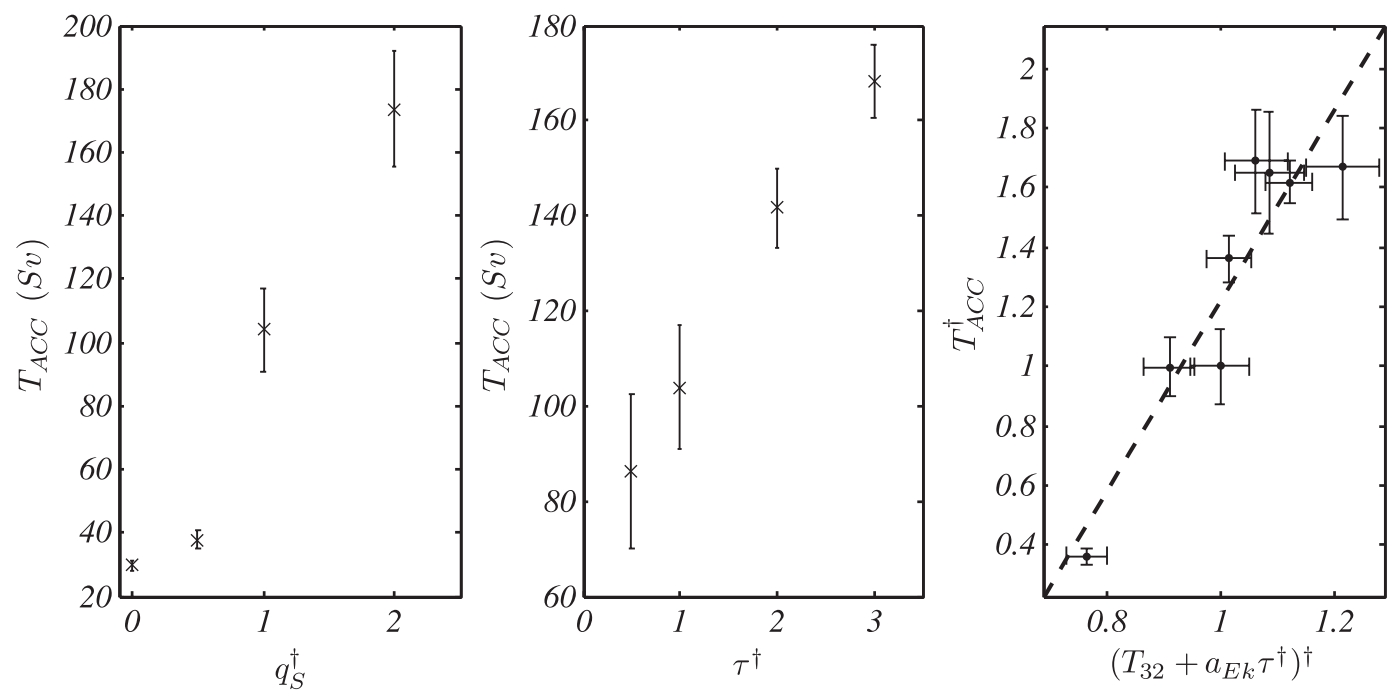

FIG. 8. The response of the ACC transport to (left) changing southern buoyancy flux and (middle) wind stress, for reference values of the other independent forcing parameters. (right) The variation of the ACC transport with the derived scale from the analytical model is also shown. The values of $T_{\mathrm{ACC}}$ and uncertainty $\delta T_{\mathrm{ACC}}$ for each run are taken as the mean and standard deviation, respectively, of a 130-yr average of the ACC transport. The uncertainty in $T_{32}$ is computed as $\delta T_{32} \approx 2 \delta h_{L} T_{32} / h_{2}$, as described previously.

transports from the analytical model using the parameterizations of section 2 ,

$$
\begin{aligned}
T_{\mathrm{ACC}} & \sim \frac{1}{2\left|f_{\mathrm{ACC}}\right|}\left(\Delta b_{13} h_{1}^{2}+\Delta b_{23} h_{2}^{2}\right) \\
& =\frac{1}{2\left|f_{\mathrm{ACC}}\right|}\left(\frac{T_{\mathrm{Ek}}-T_{31}}{a_{e, 1}}+\frac{T_{23}}{a_{e, 2}}\right) \\
& =\frac{1}{2\left|f_{\mathrm{ACC}}\right| a_{e}}\left(T_{\mathrm{Ek}}+T_{32}\right) \text { for } a_{e, 1}=a_{e, 2}=a_{e} .
\end{aligned}
$$

The final simplification has been made by approximating that the eddy scaling constants are equal and then applying the layer-3 volume balance [Eq. (7b)]. Thus, we find that the ACC transport is linear with (i) the Ekman transport $T_{\mathrm{Ek}}=a_{\mathrm{Ek}} \tau^{\dagger}$ and (ii) the abyssal overturning $T_{32}$ and thus corresponds to the sum of a wind only and coupled wind/buoyancy forcing term. This two component scaling is shown in Fig. 8 using a reference Ekman transport of $a_{\mathrm{Ek}}=2 \mathrm{~Sv}\left(1 \mathrm{~Sv} \equiv 10^{6} \mathrm{~m}^{3} \mathrm{~s}^{-1}\right)$ and is accurate to within the estimated uncertainties. The response of $T_{\mathrm{ACC}}$ to changed $q_{S}$ and $\tau$ may be explained in terms of this scaling as follows: As seen in section $4 \mathrm{c}, T_{32}$ is almost entirely controlled by the buoyancy difference $\Delta b_{13}$, and the effect of $q_{\mathrm{S}}$ is to increase this buoyancy difference. Therefore, the ACC transport increases with $q_{s}$. In terms of the wind forcing, Eq. (33) implies that increased surface wind stress results in two competing effects on the ACC transport. First, the increased Ekman transport increases the upper-ocean volume (and thus layer thicknesses $h_{1}$ and $h_{2}$ ) at the expense of the abyssal ocean and therefore also increases isopycnal slopes and the ACC transport. Second, the increased Ekman transport mixes more dense abyssal water into the upper ocean, thereby reducing $\Delta b_{13}$ and thus the ACC transport, and this effect enters Eq. (33) through the $T_{32}$ term. The near-linear trend of $T_{\mathrm{ACC}}$ with wind stress observed in Fig. 8 indicates that the first effect must dominate, at least for the wind stress magnitudes and reference buoyancy forcing used in the numerical model.

\section{e. Sensitivity to equilibration}

It was argued in section 3 that the qualitative aspects of the results are insensitive to the value of the equilibration threshold used in the numerical model. To illustrate this point, we select the most sensitive field to equilibration, the ACC transport, and compare the forcing response that is observed at various stages of model spinup. In Fig. 9, we display the response of the ACC transport to buoyancy and wind variations at the equilibration time (as in the two left-hand panels of Fig. 8) and at fractions of 0.6 and 0.8 of this time. At both lesser times, the measured transports are generally outside the error bounds for the fully equilibrated case, but the trends with forcing (shown by lines of best fit) are similar. Thus, although the magnitude of the forcing response depends upon the chosen equilibration threshold, the qualitative nature of the response is unchanged. 

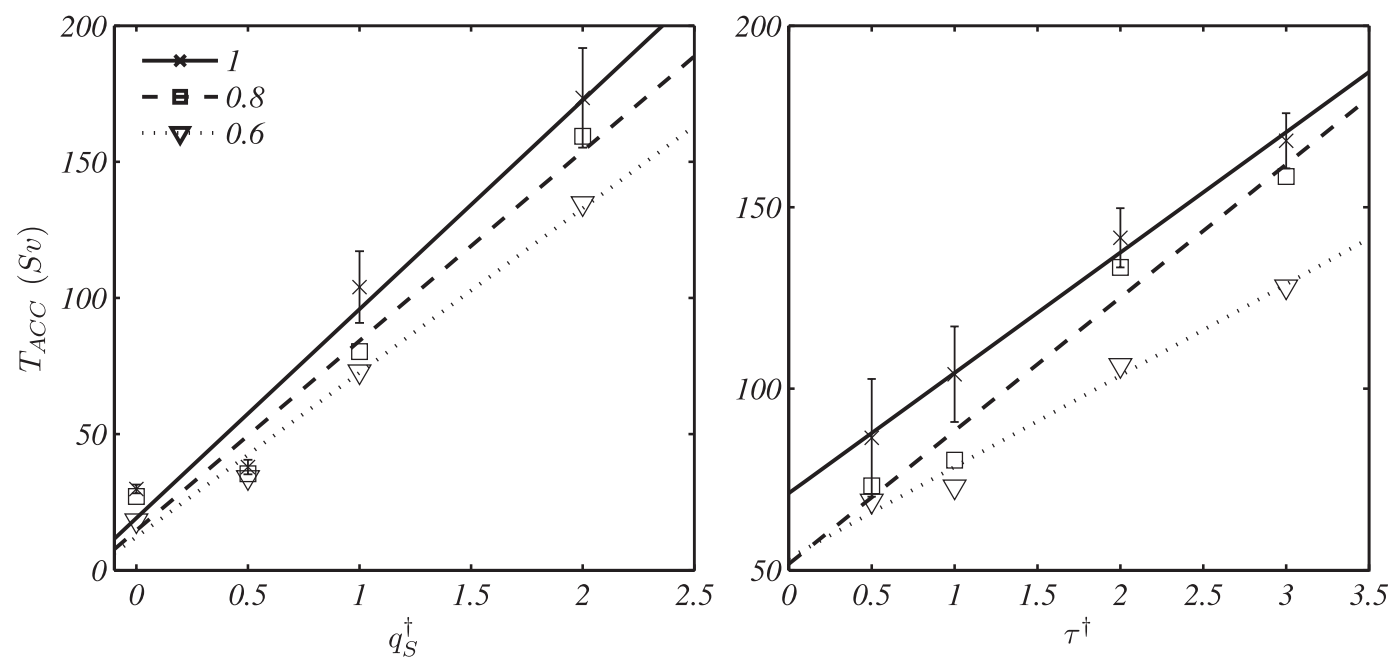

FIG. 9. The sensitivity of the forcing response of the ACC transport to equilibration threshold. The response of transport to (left) southern buoyancy flux and (right) wind stress are shown for fractions of $0.6,0.8$, and 1.0 of the equilibration time. The trends at each time are displayed by lines of best fit.

\section{Discussion}

We have presented an analytical model of the meridional overturning circulation, its associated stratification, and the Antarctic Circumpolar Current, applicable to an idealized Atlantic basin. Our model is distinct from previous analytical models in that it is based on a three-layer structure with the layers determined by the depths of overturning extrema, thus allowing the description of both the North Atlantic and abyssal cells of the MOC. The model explicitly includes surface buoyancy forcing via prescribed surface fluxes in the thermodynamic balance for each layer. Although difficulties in parameterizing diffusive fluxes prevent the formulation of a general solution, we obtain scales for the layer thicknesses and buoyancies in the adiabatic limit. These scales accurately describe the results of an eddy-permitting general circulation model with an explicit low vertical diffusivity of $10^{-5} \mathrm{~m}^{2} \mathrm{~s}^{-1}$ and minimized numerical diffusion.

A number of key results emerge from our analytical model. First, Southern Ocean wind stress and surface buoyancy forcing are of equal importance in controlling both the stratification and circulation of the ocean. The stratification and circulation are linked by the thermodynamic balance for each layer. Winds drive circulation and then alter the stratification in order to maintain thermodynamic equilibrium, whereas buoyancy forcing strongly controls the stratification and then must alter the circulation for the same reason. Negative surface buoyancy fluxes near Antarctica exert strong control over the entire ocean and should not be omitted in an analytical model. These fluxes lead to the formation of a dense abyssal layer, which, because of the thermodynamic balance for the layer, must interact with the upper ocean even in the adiabatic limit. The requirement of thermodynamic equilibrium thus leads to a coupling of the upper and abyssal ocean and interdependence of the two MOC cells.

The analytical model was also employed to derive scales for the ACC transport and overturning magnitudes, which show good agreement with the numerical simulations. The ACC transport scale may be expressed as the sum of a wind only term (the Ekman transport) and a coupled wind-buoyancy term (the abyssal overturning). In the limit of zero wind, the Ekman transport vanishes, but buoyancy fluxes still drive an abyssal overturning and thus a finite ACC transport exists. Similarly, in the limit of zero buoyancy forcing the abyssal overturning vanishes, but winds still drive an Ekman flux and thus a finite ACC transport. This result is incompatible with the theory that the ACC is eddy saturated and thus invariant to changes in wind stress, as proposed by Hallberg and Gnanadesikan (2006), Meredith and Hogg (2006), and others. We find that, although the surface wind stresses over the Southern Ocean do amplify the mesoscale eddy field, they also have a long-time-scale effect on the global stratification [as proposed by Borowski et al. (2002) and Jones et al. (2011)] and therefore strong control over the equilibrium ACC transport via the thermal wind relation. Like the ACC transport, the overturning magnitudes are dependent on both wind and buoyancy forcing. The North Atlantic overturning is found to scale linearly with both the Southern Ocean wind stress and northern buoyancy 
flux. The abyssal overturning scales inversely to the North Atlantic overturning; it decreases with increasing wind stress and increases for greater southern buoyancy flux.

There are a number of caveats on this study. We chose to describe the surface buoyancy forcing completely in terms of a prescribed flux when in fact the oceanatmosphere buoyancy exchange is a combination of relaxation and fixed-flux processes. We also did not distinguish between heat and salt in the thermodynamic balance and thus did not allow the possibility of multiple equilibria (e.g., Johnson et al. 2007). Additionally, the analytical model was only solved in the adiabatic limit, and the scaling predictions were confirmed in the numerical model for a single diffusivity value. We do not know (and cannot predict) what effect altering the diffusion would have on the results. That said, the analytical framework is open to an extension to the fully diabatic case if appropriate parameterizations for the diffusive fluxes can be found. Finally, as always, the parameter dependence arising from the analytical model is dependent on the form of the parameterization of mesoscale eddies. However, unlike almost all previous models (e.g., G99; Johnson et al. 2007; Nikurashin and Vallis 2011, 2012), we have not used the more common Gent-McWilliams (GM) scaling (Gent and McWilliams 1990; Gent et al. 1995) but the improved scheme by Visbeck et al. (1997), and this likely explains the good agreement with the eddy-permitting simulations.

Acknowledgments. We thank Adele Morrison and Henk Dijkstra for their discussions and comments that have improved this manuscript. Additionally, we are grateful to our three anonymous reviewers for helping to improve the clarity and rigor of the manuscript. This work was conducted while CJS was studying Honours at RSES, with the support of an A. L. Hales Scholarship. Both authors were supported by the ARC Centre of Excellence for Climate System Science (CE11E0098). This research was undertaken on the NCI National Facility in Canberra, Australia, which is supported by the Australian Commonwealth Government.

\section{REFERENCES}

Allison, L. C., H. L. Johnson, and D. P. Marshall, 2011: Spin-up and adjustment of the Antarctic Circumpolar Current and global pycnocline. J. Mar. Res., 69, 167-189, doi:10.1357/ 002224011798765330.

Borowski, D., R. Gerdes, and D. Olbers, 2002: Thermohaline and wind forcing of a circumpolar channel with blocked geostrophic contours. J. Phys. Oceanogr., 32, 2520-2538. de Boer, A. M., A. Gnanadesikan, N. R. Edwards, and A. J. Watson, 2010: Meridional density gradients do not control the Atlantic overturning circulation. J. Phys. Oceanogr., 40, 368-380.

Fučkar, N. S., and G. K. Vallis, 2007: Interhemispheric influence of surface buoyancy conditions on a circumpolar current. Geophys. Res. Lett., 34, L14605, doi:10.1029/2007GL030379.

Gent, P. R., and J. C. McWilliams, 1990: Isopycnal mixing in ocean circulation models. J. Phys. Oceanogr., 20, 150-155.

_ J. Willebrand, T. J. McDougall, and J. C. McWilliams, 1995: Parameterizing eddy-induced tracer transports in ocean circulation models. J. Phys. Oceanogr., 25, 463-474.

Gnanadesikan, A., 1999: A simple predictive model for the structure of the oceanic pycnocline. Science, 283, 2077-2079.

Gordon, A., 2009: Bottom water formation. Ocean Currents: A Derivative of the Encyclopedia of Ocean Sciences, J. H. Steele, S. A. Thorpe, and K. K. Turekian, Eds., Academic Press, 263-269.

Hallberg, R., and A. Gnanadesikan, 2006: The role of eddies in determining the structure and response of the wind-driven Southern Hemisphere overturning: Results from the Modeling Eddies in the Southern Ocean (MESO) project. J. Phys. Oceanogr., 36, 2232-2252.

Hogg, A. M., 2010: An Antarctic Circumpolar Current driven by surface buoyancy forcing. Geophys. Res. Lett., 37, L23601, doi:10.1029/2010GL044777.

Hughes, G. O., and R. W. Griffiths, 2006: A simple convective model of the global overturning circulation, including effects of entrainment into sinking regions. Ocean Modell., 12, 46-79.

Ilicak, M., A. J. Adcroft, S. M. Griffies, and R. W. Hallberg, 2012: Spurious dianeutral mixing and the role of momentum closure. Ocean Modell., 45-46, 37-58.

Ito, T., and J. Marshall, 2008: Control of lower-limb overturning circulation in the Southern Ocean by diapycnal mixing and mesoscale eddy transfer. J. Phys. Oceanogr., 38, 28322845.

Johnson, H. L., D. P. Marshall, and D. A. J. Sproson, 2007: Reconciling theories of a mechanically driven meridional overturning circulation with thermohaline forcing and multiple equilibria. Climate Dyn., 29, 821-836.

Jones, D. C., T. Ito, and N. S. Lovenduski, 2011: The transient response of the Southern Ocean pycnocline to changing atmospheric winds. Geophys. Res. Lett., 38, L15604, doi:10.1029/ 2011 GL048145.

Killworth, P. D., 2001: On the rate of descent of overflows. J. Geophys. Res., 106, 22 267-22 275.

Levermann, A., and A. Griesel, 2004: Solution of a model for the oceanic pycnocline depth: Scaling of overturning strength and meridional pressure difference. Geophys. Res. Lett., 31, L17302, doi:10.1029/2004GL020678.

_ , and J. J. Furst, 2010: Atlantic pycnocline theory scrutinized using a coupled climate model. Geophys. Res. Lett., 37, L14602, doi:10.1029/2010GL044180.

Lumpkin, R., and K. Speer, 2007: Global ocean meridional overturning. J. Phys. Oceanogr., 37, 2550-2562.

Marshall, J., and T. Radko, 2003: Residual-mean solutions for the Antarctic Circumpolar Current and its associated overturning circulation. J. Phys. Oceanogr., 33, 2341-2354.

_ A. Adcroft, C. Hill, L. Perelman, and C. Heisey, 1997: A finite volume, incompressible Navier Stokes model for studies of the ocean on parallel computers. J. Geophys. Res., 102, 57535766. 
Meredith, M. P., and A. M. Hogg, 2006: Circumpolar response of Southern Ocean eddy activity to a change in the Southern Annular Mode. Geophys. Res. Lett., 33, L16608, doi:10.1029/ 2006 GL026499.

Nikurashin, M., and G. Vallis, 2011: A theory of deep stratification and overturning circulation in the ocean. J. Phys. Oceanogr., 41, 485-502.

_ and - 2012: A theory of the interhemispheric meridional overturning circulation and associated stratification. J. Phys. Oceanogr., in press.

Rintoul, S. R., C. W. Hughes, and D. Olbers, 2001: The Antarctic Circumpolar Current system. Ocean Circulation and Climate: Observing and Modelling the Global Ocean, G. Siedler, J. Church, and J. Gould, Eds., Academic Press, 271-302.

Samelson, R. M., 2009: A simple dynamical model of the warmwater branch of the middepth meridional overturning cell. J. Phys. Oceanogr., 39, 1216-1230.
— 2011: Time-dependent adjustment in a simple model of the middepth meridional overturning cell. J. Phys. Oceanogr., 41, 1009-1025.

_ , and G. K. Vallis, 1997: Large-scale circulation with small diapycnal diffusion: The two-thermocline limit. J. Mar. Res., $\mathbf{5 5}, 223-275$

Sloyan, B. M., and S. R. Rintoul, 2001: Circulation, renewal, and modification of Antarctic Mode and Intermediate Water. J. Phys. Oceanogr., 31, 1005-1030.

Toggweiler, J. R., and R. M. Samelson, 1995: Effect of Drake Passage on the global thermohaline circulation. Deep-Sea Res., 42, 447-500.

Visbeck, M., J. Marshall, T. Haine, and M. A. Spall, 1997: Specification of eddy transfer coefficients in coarse-resolution ocean circulation models. J. Phys. Oceanogr., 27, 381-402.

Wolfe, C. L., and P. Cessi, 2011: The adiabatic pole-to-pole overturning circulation. J. Phys. Oceanogr., 41, 1795-1810. 